Clinical Research Paper

\title{
Metastatic to negative Iymph node ratio demonstrates significant prognostic value in patients with esophageal squamous cell carcinoma after esophagectomy
}

\author{
Xiao-Feng Duan ${ }^{1}$, Peng Tang ${ }^{1}$, Xiao-Bin Shang ${ }^{1}$, Hong-Jing Jiang ${ }^{1}$ and Zhen-Tao Yu ${ }^{1}$ \\ ${ }^{1}$ Department of Esophageal Cancer, Tianjin Medical University Cancer Institute and Hospital, National Clinical Research \\ Center of Cancer, Key Laboratory of Cancer Prevention and Therapy of Tianjin City, Clinical Research Center for Cancer of \\ Tianjin City, Tianjin, China \\ Correspondence to: Zhen-Tao Yu, email: yuzhtao@hotmail.com \\ Keywords: esophageal squamous cell carcinoma, esophagectomy, lymph node ratio, MNLNR, prognosis \\ Received: April 07, $2017 \quad$ Accepted: May 16, $2017 \quad$ Published: July 28, 2017 \\ Copyright: Duan et al. This is an open-access article distributed under the terms of the Creative Commons Attribution License 3.0 \\ (CC BY 3.0), which permits unrestricted use, distribution, and reproduction in any medium, provided the original author and source \\ are credited.
}

\section{ABSTRACT}

Aims: The prognostic value of metastatic lymph node ratio (LNR) has been reported in some studies; however, there is no report on the prognostic significance of metastatic to negative lymph node ratio (MNLNR) in cancer patients. The aim of this study was to compare the prognostic value of $p N$, LNR and MNLNR on the survival of patients with esophageal squamous cell carcinoma (ESCC) after esophagectomy.

Methods: The data of $\mathbf{2 9 0}$ patients with ESCC after curative esophagectomy was retrospectively analyzed. The optimal cut-point for LNR and MNLNR were set as 0 , 01-0.2, and $>0.2$. Univariate and multivariate analyses were performed to identify prognostic factors for overall survival (OS).

Results: Patients classified as LNR 0, 0.01-0.20, and 0.21-1.0, the observed 5 -year OS rates were $46.6 \%, 26.0 \%$, and $11.6 \%$, respectively $(P=0.000)$. Patients classified as MNLNR 0, 0.01-0.20, and $>0.2$, the observed 5-year OS rates were $46.6 \%$, $31.2 \%$, and $7.4 \%$, respectively, respectively $(P=0.000)$. The pN stage, LNR or MNLNR category was confirmed as a significant independent prognostic factor, respectively $(P$ $=0.032, P=0.011$ and $P=0.003$, respectively); However, only the MNLNR category $(P=0.003)$ remained as a significant prognostic factor when the pN stage, LNR and MNLNR category simultaneously included in the multivariate analysis models.

Conclusions: The MNLNR was recognized as an independent prognostic factor in ESCC patients after curative esophagectomy. In addition, MNLNR showed better prognostic value than $\mathrm{pN}$ stage and LNR category.

\section{INTRODUCTION}

Esophageal cancer (EC) is the eighth most common cancer and the sixth most common cause of cancerrelated deaths worldwide [1]. In 2012, there were an estimated 455,800 new EC cases and 400,200 associated deaths worldwide [1]. In China, there were an estimated 286,700 new EC cases and 210,900 associated deaths [2]. The prognosis of patients with EC remains poor with 5 -year OS rates of $15 \%-25 \%$ for all EC patients [3] The two main types of EC are squamous cell carcinoma and adenocarcinoma. In high-risk area, including China, esophageal squamous cell carcinoma (ESCC) accounts for $>90 \%$ of all EC cases [1]. Surgical resection is still the main treatment option for ESCC patients. Despite a marked reduction in surgical morbidity and mortality and the application of comprehensive treatment, the long-term survival of esophageal cancer patients after surgery remains poor. This poor prognosis has prompted us to investigate more effective prognostic factors for long-term survival.

Lymph node metastasis is an important prognostic factor and indicator for subsequent adjuvant treatments in EC patients. However, a small number of removed lymph nodes (LNs) may lead to under-staging and subsequent 
under-estimation of disease severity, which is referred to as stage migration [4]. Lymph node ratio (LNR), which is calculated as the ratio of the number of metastatic LNs to the total number of removed LNs, has been proposed to address the problems related to the variability of nodal examination. It has been confirmed as a highly reliable indicator used to evaluate the prognosis of cancer patients. The advantage of LNR over the $7^{\text {th }}$ American Joint Committee on Cancer (AJCC) N category in predicting the prognosis of ESCC patients has been explored in some studies [5-15].

A recent study also showed that the number of negative lymph nodes (NLNs) could impact the OS of patients with ESCC, especially among those with nodal-positive disease and advanced T-stage tumor [16]. The prognostic value of NLNs was also confirmed in previous studies that revealed that a higher number of NLNs was associated with better OS in EC patients after esophagectomy [17-18]. Metastatic to NLNs ratio (MNLNR), which is calculated as the ratio of the number of metastatic LNs to the NLNs, has not been proposed to evaluate its prognostic value in any cancer patients after curative resection. In the light of these considerations, our study was conducted to 1) show whether MNLNR could address the problems related to the variability of nodal examination and stage migration, 2) determine the prognostic value and the relation of MNLNR with OS, 3) compare MNLNR with $\mathrm{pN}$ stage and LNR for the prognostic evaluation of ESCC after curative esophagectomy.

\section{RESULTS}

\section{Patient demographics}

The clinicopathological parameters of the 290 ESCC patients included in our study were summarized in Table 1. There were 238 males and 52 females with a median age of 68 years (range, 35-95 years). According to histological grade, 19 patients were well differentiated, 240 were moderately differentiated, and 31 were poorly differentiated. In the cohort, the average number of total retrieved LNs per patient was 15.2 (range, 4-58). According to the 7 th edition AJCC TNM staging system, $172,71,30$, and 10 patients were classified as N0, N1, $\mathrm{N} 2$, and N3, respectively. As regard to the TNM staging system, 17 patients were in stage I, 104 patients were in stage II, 155 patients were in stage III, and seven patients were in stage IV.

\section{Correlation of the number of retrieved nodes to metastatic nodes, LNR and MNLNR}

Spearman's correlation analysis showed that the total number of retrieved LNs was signifcantly related to the number of metastatic LNs ( $\mathrm{r}=0.168, P=0.005$; Figure 1A), whereas the number of retrieved LNs was not correlated with LNR $(\mathrm{r}=0.041, P=0.491$; Figure $1 \mathrm{~B})$ and MNLNR ( $\mathrm{r}=0.068, \mathrm{P}=0.254$; Figure 1C).

\section{The optimal cut-point value of LNR and MLNR}

The patients without lymph node metastasis were classifed as LNR 0. Furthermore, the other patients were stratified into five groups by every 0.20 interval of LNR. There were only 13 patients with a LNR value of $0.41-1$, and the 13 patients were grouped as $>0.4$. According to the best cut-off approach by the log-rank test, the survival rates for the categories 0.21-0.4 and $>0.4$ were similar. So we divided the LNR into three subgroups as follows: LNR, $0(\mathrm{n}=172)$; LNR, 0.01-0.2 $(\mathrm{n}=72)$; LNR, >0.2 $(\mathrm{n}=39)$.

The patients without lymph node metastasis were classifed as MNLNR 0. Furthermore, the other patients were stratified into five groups by every 0.20 interval of MNLNR. There were only 23 patients with a MNLNR value of $>0.4$, and the 23 patients were grouped as $>0.4$. According to the best cut-off approach by the log-rank test, the survival rates for the categories 0.21-0.4 and > 0.4 were similar. So we divided the MNLNR into three subgroups as follows: MNLNR, $0(\mathrm{n}=172)$; MNLNR, 0.01-0.2 $(\mathrm{n}=65) ; \operatorname{MNLNR},>0.2(\mathrm{n}=46)$.

\section{Univariate survival analysis}

The 1, 3, 5-year OS rates of the entire cohort were $81.0 \%, 62.0 \%$ and $37.3 \%$, respectively. For patients classified as N0, N1, N2, and N3 according to the AJCC N category, the 5-year OS rates were $46.6 \%, 41.1 \%, 22.1 \%$ and 0 , respectively $(\chi 2=7.372, P=0.007$ by log-rank test).

Patients classified as LNR 0, LNR 0.01-0.2, and LNR 0.21 - 1 , the observed 5-year OS rates were $46.6 \%, 26.0 \%$, and $11.6 \%$, respectively, and the median survival times of these three groups were 58.0, 36.0, and 24.0 months, respectively $(\chi 2=16.158, P=0.000$ by log-rank test).

Patients classified as MNLNR 0, MNLNR 0.01-0.2, and MNLNR $>0.2$, the observed 5-year OS rates were $46.6 \%, 31.2 \%$, and $7.4 \%$, respectively, and the median survival times of these three groups were 58.0, 42.0, and 33.0 months, respectively $(\chi 2=17.375, P=0.000$ by log-rank test). The survival curves according to the $\mathrm{pN}$ category, the LNR category, and MNLNR are shown in Figure 2, Figure 3 and Figure 4.

The clinicopathological factors analyzed in the univariate survival analysis are also shown in Table 1. The factors significantly influencing the 5-year OS were sex $(\mathrm{P}=0.040)$, histological grade $(\mathrm{P}=0.044)$, $\mathrm{pT}$ stage $(\mathrm{P}=$ $0.022)$, pN stage $(\mathrm{P}=0.007)$, LNR category $(\mathrm{P}=0.000)$, and MNLNR $(\mathrm{P}=0.000)$ after esophagectomy. 
Table 1: Clinicopathological features and univariate survival analysis

\begin{tabular}{|c|c|c|c|c|c|c|}
\hline Variables & $\mathrm{n}=\mathbf{2 9 0}$ & 5-year OS (\%) & HR & $95 \% \mathrm{CI}$ & $\chi^{2}$ & $\mathbf{P}$ \\
\hline Gender & & & 0.595 & $0.358-0.988$ & 4.205 & 0.040 \\
\hline Male & 238 & 35.2 & & & & \\
\hline Female & 52 & 48.6 & & & & \\
\hline Age (years) & & & 1.326 & $0.958-1.836$ & 2.981 & 0.084 \\
\hline$<65$ & 118 & 46.5 & & & & \\
\hline$\geq 65$ & 172 & 31.3 & & & & \\
\hline Smokig history & & & 1.118 & $0.792-1.579$ & 0.417 & 0.519 \\
\hline Yes & 208 & 35.6 & & & & \\
\hline No & 82 & 41.5 & & & & \\
\hline Drinking history & & & 1.109 & $0.087-1.552$ & 0.418 & 0.518 \\
\hline Yes & 146 & 36.2 & & & & \\
\hline No & 144 & 38.8 & & & & \\
\hline Tumor location & & & 1.186 & $0.830-1.696$ & 4.642 & 0.098 \\
\hline Upper & 15 & 57.8 & & & & \\
\hline Middle & 220 & 34.8 & & & & \\
\hline Lower & 55 & 37.9 & & & & \\
\hline Tumor size & & & 1.007 & $0.712-1.446$ & 0.022 & 0.966 \\
\hline$<4 \mathrm{~cm}$ & 174 & 38.5 & & & & \\
\hline$\geq 4 \mathrm{~cm}$ & 116 & 33.8 & & & & \\
\hline Differentiation & & & 0.725 & $0.525-0.098$ & 4.060 & 0.044 \\
\hline Well & 19 & 56.0 & & & & \\
\hline Moderate & 240 & 38.0 & & & & \\
\hline Poor & 31 & 24.9 & & & & \\
\hline pT stage & & & 1.185 & $1.022-1.374$ & 5.272 & 0.022 \\
\hline $\mathrm{T} 1-2$ & 61 & 43.4 & & & & \\
\hline T3-4 & 229 & 36.0 & & & & \\
\hline pN stage* & & & 1.493 & $1.201-1.856$ & 7.372 & 0.007 \\
\hline 0 & 172 & 46.6 & & & & \\
\hline 1 & 71 & 41.1 & & & & \\
\hline 2 & 30 & 22.1 & & & & \\
\hline 3 & 10 & 0 & & & & \\
\hline pTNM stage* & & & 1.578 & $1.202-2.073$ & 19.050 & 0.000 \\
\hline I & 17 & 63.2 & & & & \\
\hline II & 104 & 42.7 & & & & \\
\hline III & 155 & 32.4 & & & & \\
\hline IV & 7 & 0 & & & & \\
\hline
\end{tabular}

(Continued) 


\begin{tabular}{|c|c|c|c|c|c|c|}
\hline Variables & $\mathrm{n}=\mathbf{2 9 0}$ & 5-year OS (\%) & HR & $95 \%$ CI & $\chi^{2}$ & $\mathbf{P}$ \\
\hline $\mathrm{LNR}^{*}$ & & & 1.451 & $1.223-1.721$ & 16.158 & 0.000 \\
\hline 0 & 172 & 46.6 & & & & \\
\hline$\sim 0.2$ & 72 & 26.0 & & & & \\
\hline$>0.2$ & 39 & 11.6 & & & & \\
\hline MNLNR* & & & 1.601 & $1.265-2.025$ & 17.375 & 0.000 \\
\hline 0 & 172 & 46.6 & & & & \\
\hline$\sim 0.2$ & 65 & 31.2 & & & & \\
\hline$>0.2$ & 46 & 7.4 & & & & \\
\hline
\end{tabular}

* Seven patients can not evaluate the $\mathrm{N}$ status; the total number of patients was 283.

Log-rank test was used to compare survival in groups. LNR, metastatic lymph node ratio; MNLNR, metastatic/negative lymph node ratio; OS, overall survival; HR, hazard ratio; CI, confidence interval.

\section{Multivariate survival analysis}

Multivariate survival analysis was performed with Cox's proportional hazard regression model to identify the independent factors correlated with prognosis. There were three different lymph node stages, pN, LNR and MNLNR in the present study. First, we put each lymph node stage into multivariate survival analysis to confirm the prognostic value of each lymph node category. Finally, we put all three stages into final analysis to confirm the value of MNLNR compared with $\mathrm{pN}$ and LNR category.

When either $\mathrm{N}$ category or LNR category or MNLNR was included in the analysis models, it was found to be one of the most significant independent prognostic factors for OS, in addition to gender and pTNM stage $(\mathrm{P}<0.05$ for these parameters). However, the $\mathrm{N}$ category and LNR $(\mathrm{P}>0.05)$ no longer significantly predicted survival when the $\mathrm{N}$ category, the LNR category, and the MNLNR were simultaneously considered covariates. By comparison, the MNLNR category $(\mathrm{P}=0.003)$ remained as a significant indicator of prognosis (Table 2).

\section{DISCUSSION}

The LNR has been shown to have better prognostic value than $\mathrm{pN}$ stage in $\mathrm{EC}$ patients [5-15, 19-23]. Greenstein et al. [22] used the SEER database to evaluate the relationship between LNR and survival among 838 esophageal patients with lymph node metastasis. They classified the patients into three groups according to the LNR $(\leq 0.2,0.21-0.5$, and $>0.5)$, and found that LNR can stratify survival better than the $\mathrm{pN}$ stage. A recent study included 387 ESCC patients receiving curative esophagectomy showed that compared with $\mathrm{N}$ stage, the
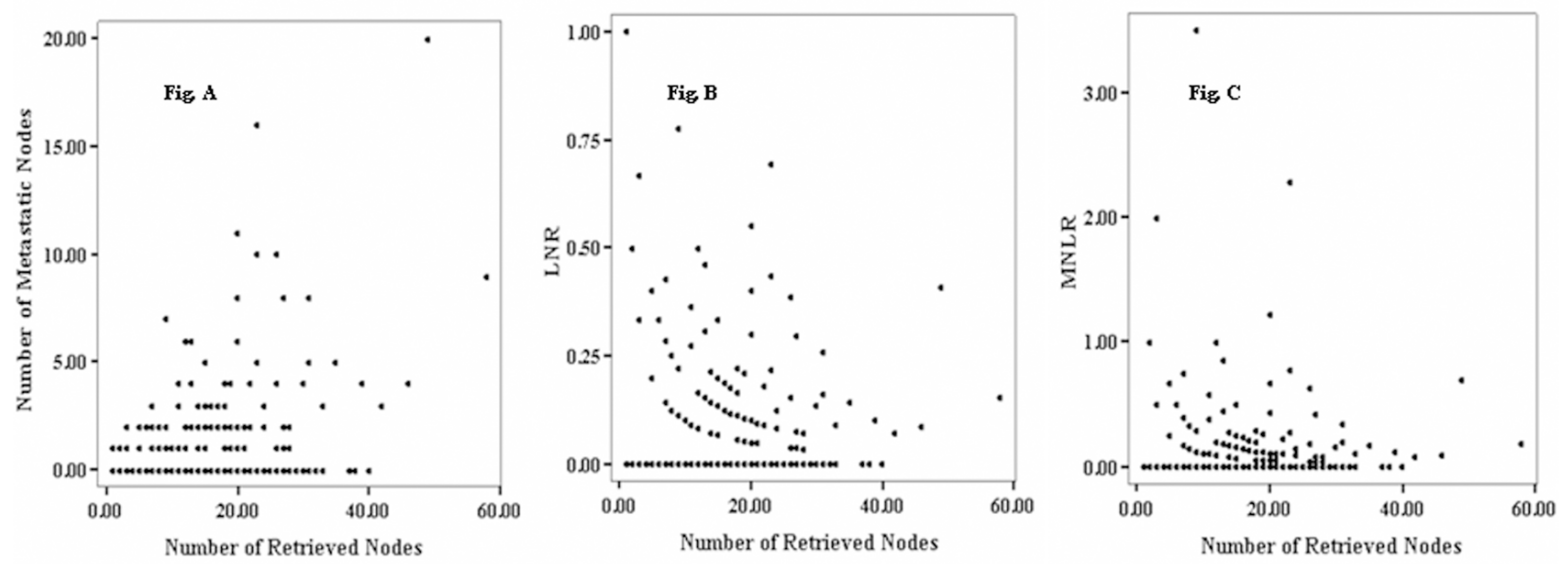

Figure 1: Spearman correlation of the number of retrieved nodes, metastatic nodes, LNR, and MNLNR. (A) Significant correlation of the number of metastatic nodes with the total number of retrieved lymph nodes $(r=0.168, P=0.005)$. (B and $\mathbf{C})$ No significant correlation of the LNR $(r=0.041, P=0.491)$ and MNLNR $(r=0.068, P=0.254)$ with the number of retrieved lymph nodes. 


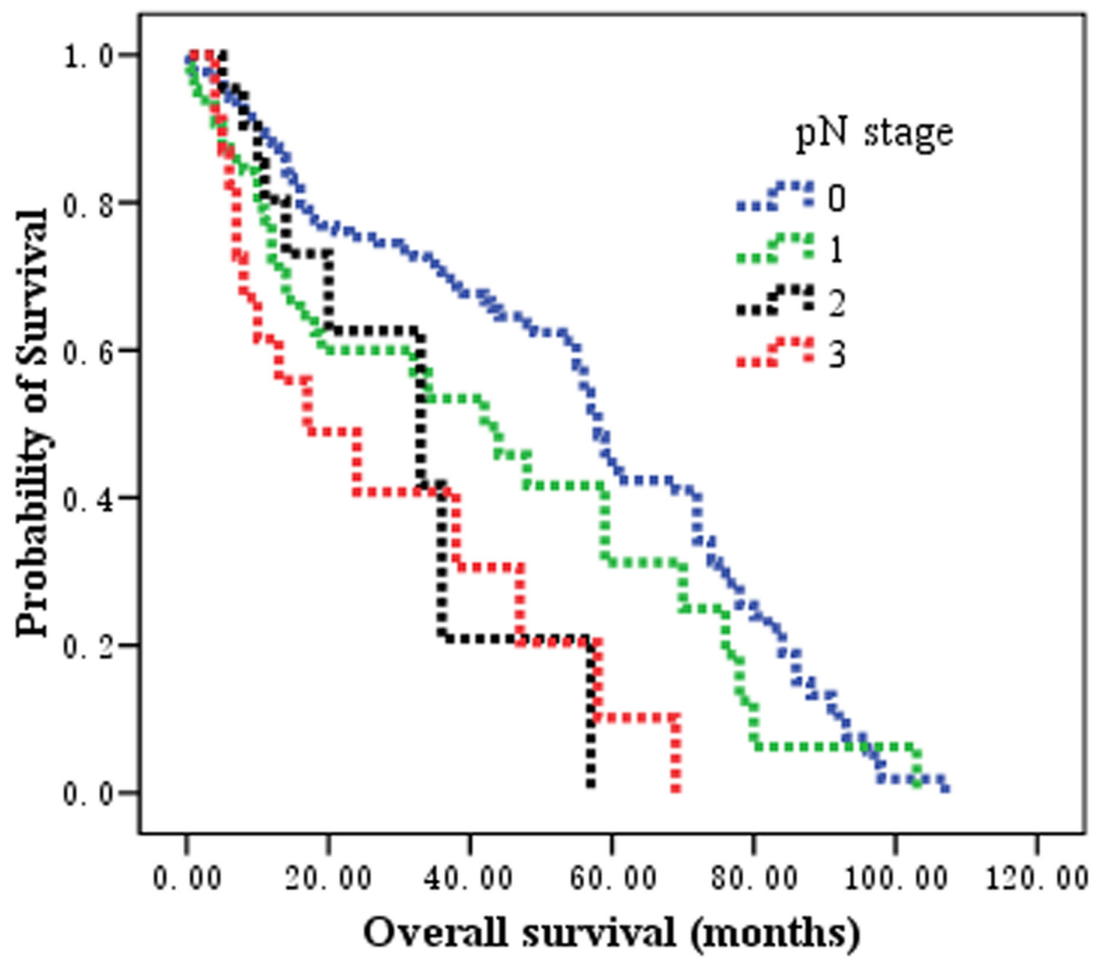

Figure 2: The prognostic significance of $\mathrm{pN}$ stage on overall survival in patients with esophageal squamous cell carcinoma after esophagectomy. Kaplan-Meier survival analysis of $\mathrm{pN}$ subgroups by log-rank test (Chi-square $=7.372, P=0.007$ ).

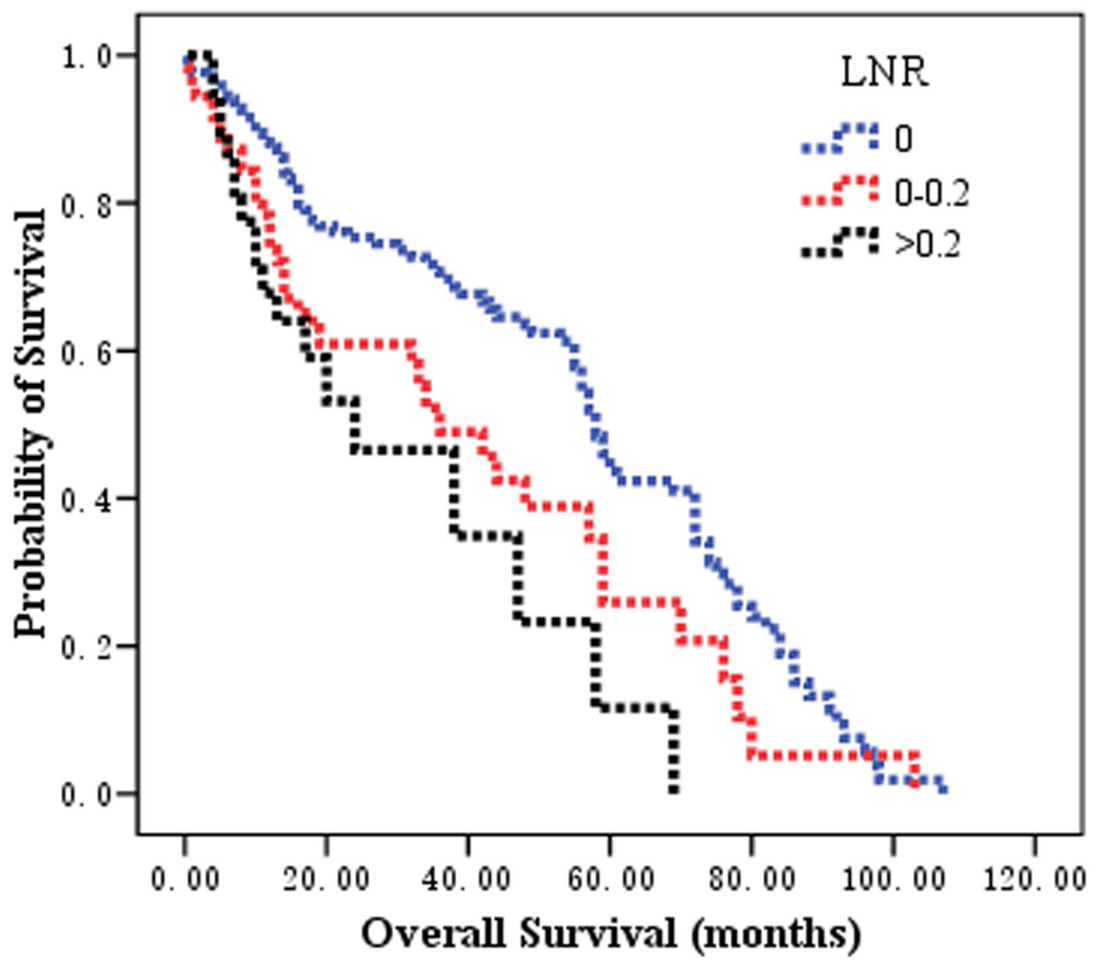

Figure 3: The prognostic significance of LNR category on overall survival in patients with esophageal squamous cell carcinoma after esophagectomy. Kaplan-Meier survival analysis of LNR subgroups by log-rank test (Chi-square $=16.158, P=0.000$ ). 
LNR stage yielded a potential superiority of the prognostic discriminatory ability and exhibited higher accuracy in determining the prognosis of patients with ESCC [14]. Recent studies showed that use of the NLNs may help to provide an accurate prognosis [17-18]. Regional LNs are the most common initial site of EC recurrence, and these nodes harbor micrometastatic disease that cannot be detected readily by standard $\mathrm{H}-\mathrm{E}$ staining techniques. Theoretically, resecting more LNs or finding more NLNs may reduce the risk of occult lesions and thus increase the survival rate.

The rationalities to propose the MNLNR were based on the following reasons. 1) The ratio of metastatic to negative lymph nodes could reflect two factors, one is the severities of lymph node metastasis (Numerator) and the other is the radicality of lymph node resection (Denominator). With more lymph node metastasis and less lymph node resection, the ratio would be bigger indicating poorer survival; with less lymph node metastasis and more lymph node resection, the ratio would be smaller indicating better prognosis. 2) The $\mathrm{pN}$ stage is affected by the number of removed lymph nodes. The positive lymph nodes may be left in the body if only a few lymph nodes were removed during the operation, which leads to an under-staged disease. LNR has been proposed to eliminate the stage migration. Whether MNLNR could address the problems related to the variability of nodal dissection and stage migration is not known. Consistent with previously reported data, our findings revealed that the number of metastatic nodes increased proportionally to the total number of dissected LNs, but the LNR and MNLNR were not correlated with the total number of retrieved LNs. These results demonstrated that MNLNR was not influenced by lymph node resection just like LNR, but $\mathrm{pN}$ was influenced by surgical procedure. 3) We established the cut-point for MNLNR on the basis of the statistical significance of OS observed with increasing values of 0.2 intervals as $0,0.01-0.20$, and $>0.20$ by performing logrank test. We found that the LNR (Figure 3) and MNLNR (Figure 4) category showed a clear advantage over the pN category (Figure 2). Our study also confirmed that MNLNR as an independent prognostic factor; still need further investigation with a bigger patient population and more detailed subgroups.

In the present study, the MNLNR category was superior to the $\mathrm{pN}$ category and LNR because of the following reasons. (i) In univariate analysis, the log-rank $\chi 2$ associated with $\operatorname{MNLNR}(\chi 2=17.375, P=0.000)$ was larger than that of the AJCC N category $(\chi 2=7.372, \mathrm{P}=$ $0.007)$ and $\operatorname{LNR}(\chi 2=16.158, P=0.000)$, indicating a higher statistical significance. (ii) In multivariate analysis, $\mathrm{pN}, \mathrm{LNR}$ or MNLNR was an independent prognostic factor for OS, respectively. However, the N stage and LNR category lost the significance when all three covariates

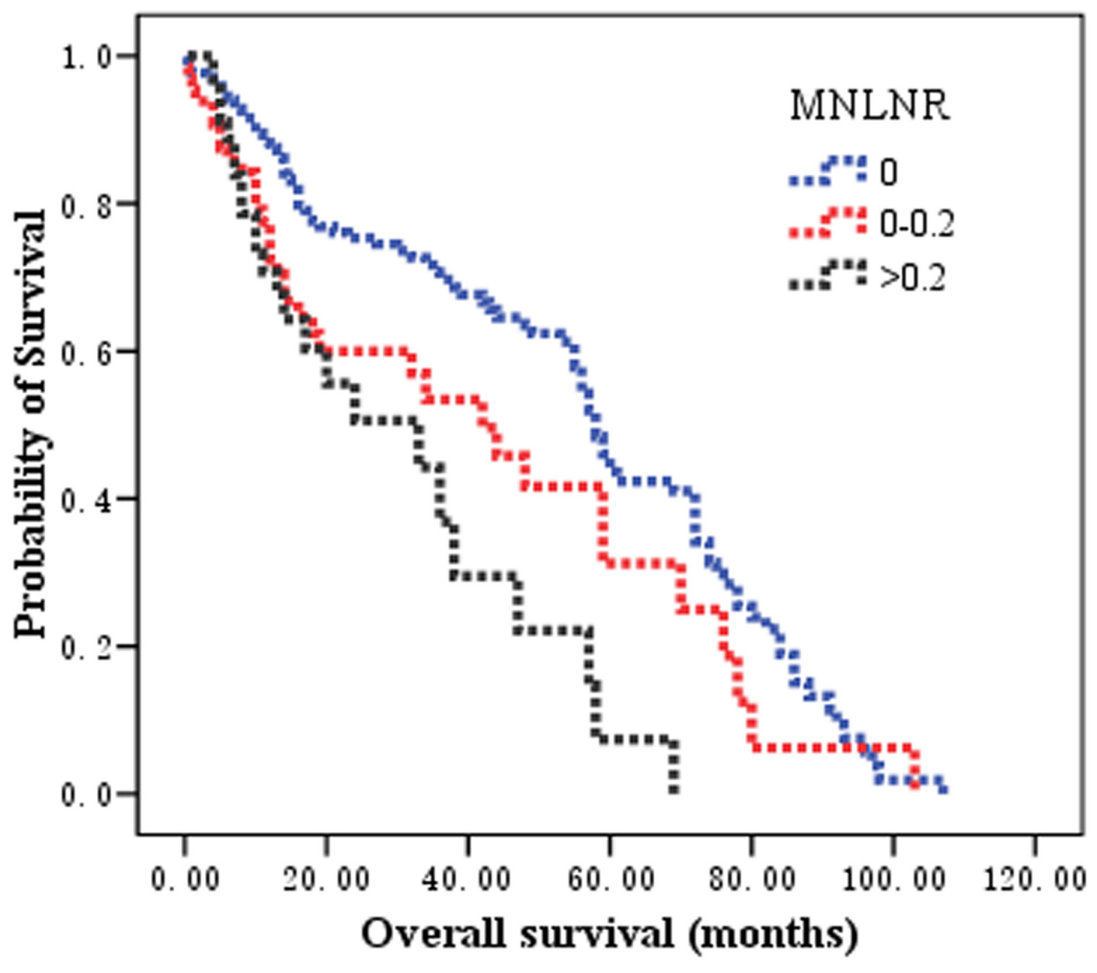

Figure 4: The prognostic significance of MNLNR category on overall survival in patients with esophageal squamous cell carcinoma after esophagectomy. Kaplan-Meier survival analysis of MNLNR subgroups by log-rank test $(\mathrm{Chi}$-square $=17.375$, $P=0.000$ ). 
Table 2: Multivariate survival analysis of the variables affecting the overall survival

\begin{tabular}{|c|c|c|c|c|c|c|c|c|c|c|c|c|}
\hline \multirow[b]{2}{*}{ Variables } & \multicolumn{3}{|c|}{ Multivariate analysis 1} & \multicolumn{3}{|c|}{ Multivariate analysis 2} & \multicolumn{3}{|c|}{ Multivariate analysis 3} & \multicolumn{3}{|c|}{ Multivariate analysis 3} \\
\hline & HR & $95 \% \mathrm{CI}$ & $\begin{array}{c}\mathbf{P} \\
\text { value }\end{array}$ & HR & $95 \% \mathrm{CI}$ & $\begin{array}{c}P \\
\text { value }\end{array}$ & HR & $95 \% \mathrm{CI}$ & $\begin{array}{c}P \\
\text { value }\end{array}$ & HR & $95 \% \mathrm{CI}$ & $\begin{array}{c}P \\
\text { value }\end{array}$ \\
\hline Gender & 0.572 & $0.343-0.952$ & 0.031 & 0.576 & $0.346-0.958$ & 0.034 & 0.570 & $0.343-0.949$ & 0.031 & 0.570 & $0.343-0.949$ & 0.031 \\
\hline $\mathrm{pN}$ & 1.316 & $1.025-1.684$ & 0.032 & - & - & - & - & - & - & 0.986 & $0.625-1.525$ & 0.792 \\
\hline pTNM & 1.438 & $1.048-1.974$ & 0.025 & 1.422 & $1.043-1.940$ & 0.026 & 1.417 & $1.045-1.922$ & 0.025 & 1.417 & $1.045-1.922$ & 0.025 \\
\hline LNR & - & - & - & 1.410 & $1.081-1.840$ & 0.011 & - & - & - & 0.692 & $0.267-1.789$ & 0.412 \\
\hline MNLNR & - & - & - & - & - & - & 1.342 & $1.108-1.625$ & 0.003 & 1.342 & $1.108-1.625$ & 0.003 \\
\hline
\end{tabular}

LNR, metastatic lymph node ratio; MNLNR, the ratio of the number of metastatic to negative lymph node; HR, hazard ratio; CI, confidence interval.

were put into the multivariate analysis and compared together (Table 2).

Several limitations should be considered in this study. Firstly, this was a retrospective study at a single cancer center. Secondly, there were only three groups based on the cut-off value of MNLNR for the relatively small patient number. It will be necessary to confirm the additional cut-off value especially for patients with MNLNR $>0.2$ and to explore the optimal cut-off point. Thirdly, it has been demonstrated that the station of the positive nodes and the number of station removed are important independent prognostic factor affecting long-term survival in patients with EC. However, the relationship between the MNLNR and the location of the positive LNs and the number of station were not investigated in our study. Finally, one of the limitations of this study is the population; maybe other studies with other populations (Africa, America, and Europe) will be needed.

In conclusion, our investigation demonstrated that the MNLNR category may be a potentially convenient and reproducible prognostic variable to reduce stage migration. The MNLNR should be considered as an independent prognostic factor in ESCC patients after curative esophagectomy. In addition, MNLNR showed a better prognostic value than $\mathrm{pN}$ stage and LNR category.

\section{PATIENTS AND METHODS}

\section{Patients}

All patients provided written consent for their information and surgical samples to be stored at the Tianjin Medical University Cancer Center used for research. This study was approved by an independent ethics committee at the Cancer Center of Tianjin Medical University. In 2014, the Department of Esophageal Cancer of Tianjin Medical University Cancer Hospital and Institute established a database of esophagectomy cases by performing a retrospective review of patients who attended the institution. The data collected for the database included patient demographics, preoperative symptoms, comorbidities, risk factors, family history, main preoperative examination results, tumor stage and histopathologic features, follow-up and survival data. Chart reviews were performed solely by experienced clinicians and were recorded on standardized abstraction forms. Data can be extracted and analyzed according to the aims of a particular study, and the database was managed in an anonymous way before the authors accessed the data.

Selective criteria included: (a) no neoadjuvant treatment; (b) complete tumor resection; (c) negative incision margins; (d) postoperative histopathologic confirmation of squamous cell carcinoma; and (e) no perioperative mortality; (f) death due to ESCC progression and cancer-related complications; (g) with follow-up data.

\section{Esophagectomy}

All patients included in our study underwent staging with physical examination, cervical ultrasonography, upper gastrointestinal radiography, endoscopy and endoscopic ultrasound, thoracic and abdominal enhanced computed tomography (CT) and/or positron emission tomography (PET) or PET-CT scans. The surgical approach was based on tumor location, tumor stage, and surgeon preference. All patients underwent transthoracic esophagectomy, and two-field or three-field lymph node dissection (if suspicious for cervical lymph node metastasis). Reconstruction was performed with a gastric tube and a thoracic or left cervical esophagogastrostomy. Pathologic stage was determined according to the 7th edition AJCC staging system [24].

\section{Lymph node classifications}

Lymph node metastasis was classified according to the 7th edition AJCC N category based on the number of metastatic lymph nodes: N0, no metastasis; N1, 1 2 metastatic LNs; N2, 3 6 metastatic LNs; and N3, $\geq 7$ metastatic LNs. In the present study, our analysis was conducted as follows to determine the appropriate cutpoint of LNR and MNLNR that determines the greatest actuarial survival difference among subgroups. Patients 
without LNs metastasis were initially assigned to one group because their prognoses significantly differed from patients with lymph node metastasis. The intervals of LNR and MNLNR categories were subsequently determined by comparing the OS rates on the basis of an initial interval of 0.2 and then combining the neighborhood survival curves by using the log-rank test [14].

\section{Follow-up}

The patients attended the institution between 2005 and 2008 were retrospectively reviewed. After curative resection, the patients were followed up according to our standard protocol: every three months for the first two years, every six months during the third to the fifth year, and then annually thereafter until death or the last followup. Clinical, laboratory, and imaging examinations were performed in each visit. Endoscopic examinations were performed when necessary. The median follow-up period after surgery for the entire cohort was 30 months (range, $3 \sim 108$ months). OS was calculated as the time from operation to the date of death or final follow-up.

\section{Statistical analysis}

Statistical analyses were performed using the SPSS software package (SPSS Standard version 18.0; SPSS, Chicago, IL, USA). Statistical analyses included univariate analyses using the $\chi 2$ or Fisher's exact tests for categorical data. Continuous variables were analyzed using ANOVA. Survival analyses were performed using Kaplan-Meier curves with log rank tests for significance. Multivariate survival analyses were performed using the Cox proportional hazard regression model. All statistical tests were two-sided and $\mathrm{P}$ value less than 0.05 was considered significant.

The variates for univariate analysis included gender (male or female), age ( $<65$ years or $\geq 65$ years), smoking and drinking history (yes or no), tumor location (upper, middle, lower), tumor size ( $<4 \mathrm{~cm}$ or $\geq 4 \mathrm{~cm})$, histological grade (well, or moderate and poor differentiation), $\mathrm{pT}$ category (T1-2 or T3-4), pN category (N0, N1, N2, or N3), pTNM stage (I, II, III, or IV), LNR category (0, 0.01-0.20, or $0.21-1.0)$, MNLNR category $(0,0.01-0.20,>0.2)$. Only those variates with $\mathrm{P}$ value less than 0.05 in univariate analysis were underwent multivariate survival analyses.

\section{CONFLICTS OF INTEREST}

The authors declare no conflicts of interest.

\section{REFERENCES}

1. Torre LA, Bray F, Siegel RL, Ferlay J, Lortet-Tieulent J, Jemal A. Global cancer statistics, 2012. CA Cancer J Clin. 2015; 65:87-108. https://doi.org/10.3322/caac.21262.
2. Chen W, Zheng R, Zuo T, Zeng H, Zhang S, He J. National cancer incidence and mortality in China, 2012. Chin J Cancer Res. 2016; 28:1-11. https://doi.org/10.3978/j. issn.1000-9604.2016.02.08.

3. Pennathur A, Gibson MK, Jobe BA, Luketich JD. Oesophageal carcinoma. Lancet. 2013; 381:400-12. https:// doi.org/10.1016/S0140-6736(12)60643-6.

4. Chandarana M, Jiwnani S, Karimundackal G, Pramesh CS. Lymphadenectomy in esophageal cancer: the real issues. Ann Thorac Surg. 2014; 98:389-90. https://doi. org/10.1016/j.athoracsur.2014.01.040.

5. Liu YP, Ma L, Wang SJ, Chen YN, Wu GX, Han M, Wang XL. Prognostic value of lymph node metastases and lymph node ratio in esophageal squamous cell carcinoma. Eur J Surg Oncol. 2010; 36:155-9. https://doi.org/10.1016/j. ejso.2009.09.005.

6. Hou X, Wei JC, Xu Y, Luo RZ, Fu JH, Zhang LJ, Lin P, Yang HX. The positive lymph node ratio predicts longterm survival in patients with operable thoracic esophageal squamous cell carcinoma in China. Ann Surg Oncol. 2013; 20:1653-9. https://doi.org/10.1245/s10434-012-2794-4.

7. Bhamidipati CM, Stukenborg GJ, Thomas CJ, Lau CL, Kozower BD, Jones DR. Pathologic lymph node ratio is a predictor of survival in esophageal cancer. Ann Thorac Surg. 2012; 94:1643-51. https://doi.org/10.1016/j. athoracsur.2012.03.078.

8. Wang N, Jia Y, Wang J, Wang X, Bao C, Song Q, Tan B, Cheng Y. Prognostic significance of lymph node ratio in esophageal cancer. Tumour Biol. 2015; 36:2335-41. https:// doi.org/10.1007/s13277-014-2840-x.

9. Tan Z, Ma G, Yang H, Zhang L, Rong T, Lin P. Can lymph node ratio replace pn categories in the tumor-nodemetastasis classification system for esophageal cancer? J Thorac Oncol. 2014; 9:1214-21. https://doi.org/10.1097/ JTO.0000000000000216.

10. Wei C, Deng WY, Li N, Shen W, Zhang C, Liu JY, Luo SX. Lymph node ratio as an alternative to the number of metastatic lymph nodes for the prediction of esophageal carcinoma patient survival. Dig Dis Sci. 2015; 60:2771-6. https://doi.org/10.1007/s10620-015-3681-1.

11. Xu XL, Zheng WH, Zhu SM, Zhao A, Mao WM. The prognostic impact of lymph node involvement in large scale operable node-positive esophageal squamous cell carcinoma patients: a 10-year experience. PLoS One. 2015; 10:e0133076. https://doi.org/10.1371/journal. pone. 0133076 .

12. Chen SB, Weng HR, Wang G, Zou XF, Liu DT, Chen YP, Zhang H. Lymph node ratio-based staging system for esophageal squamous cell carcinoma. World J Gastroenterol. 2015; 21:7514-21. https://doi.org/10.3748/ wjg.v21.i24.7514.

13. Wu SG, Li FY, Zhou J, Lin Q, Sun JY, Lin HX, Guan XX, He ZY. Prognostic value of different lymph node staging methods in esophageal squamous cell carcinoma after 
esophagectomy. Ann Thorac Surg. 2015; 99:284-90. https:// doi.org/10.1016/j.athoracsur.2014.08.036.

14. Zhang H, Liang H, Gao Y, Shang X, Gong L, Ma Z, Sun $\mathrm{K}$, Tang P, Yu Z. Metastatic lymph node ratio demonstrates better prognostic stratification than $\mathrm{pN}$ staging in patients with esophageal squamous cell carcinoma after esophagectomy. Sci Rep. 2016; 6:38804. https://doi. org/10.1038/srep38804.

15. Shao Y, Geng Y, Gu W, Ning Z, Huang J, Pei H, Jiang J. Assessment of lymph node ratio to replace the $\mathrm{pN}$ categories system of classification of the TNM system in esophageal squamous cell carcinoma. J Thorac Oncol. 2016; 11:177484. https://doi.org/10.1016/j.jtho.2016.06.019.

16. Ma M, Tang P, Jiang H, Gong L, Duan X, Shang X, Yu $Z$. Number of negative lymph nodes as a prognostic factor in esophageal squamous cell carcinoma. Asia Pac J Clin Oncol. 2016. https://doi.org/10.1111/ajco.12567.

17. Hsu PK, Huang CS, Wang BY, Wu YC, Chou TY, Hsu WH. The prognostic value of the number of negative lymph nodes in esophageal cancer patients after transthoracic resection. Ann Thorac Surg. 2013; 96:995-1001. https://doi. org/10.1016/j.athoracsur.2013.04.098.

18. Zhu Z, Chen H, Yu W, Fu X, Xiang J, Li H, Zhang Y, Sun M, Wei Q, Zhao W, Zhao K. Number of negative lymph nodes is associated with survival in thoracic esophageal squamous cell carcinoma patients undergoing three-field lymphadenectomy. Ann Surg Oncol. 2014; 21:2857-63. https://doi.org/10.1245/s10434-014-3665-y.
19. Kunisaki C, Akiyama H, Nomura M, Matsuda G, Otsuka Y, Ono HA, Shimada H. Developing an appropriate staging system for esophageal carcinoma. J Am Coll Surg. 2005; 201:884-90. https://doi.org/10.1016/j.jamcollsurg.2005.07.002.

20. Dhar DK, Hattori S, Tonomoto Y, Shimoda T, Kato H, Tachibana M, Matsuura K, Mitsumoto Y, Little AG, Nagasue N. Appraisal of a revised lymph node classification system for esophageal squamous cell cancer. Ann Thorac Surg. 2007; 83:1265-72. https://doi.org/10.1016/j. athoracsur.2006.12.003.

21. Greenstein AJ, Litle VR, Swanson SJ, Divino CM, Packer S, Wisnivesky JP. Prognostic significance of the number of lymph node metastases in esophageal cancer. J Am Coll Surg. 2008; 206:239-46. https://doi.org/10.1016/j. jamcollsurg.2007.09.003.

22. Wilson M, Rosato EL, Chojnacki KA, Chervoneva I, Kairys JC, Cohn HE, Rosato FE Sr, Berger AC. Prognostic significance of lymph node metastases and ratio in esophageal cancer. J Surg Res. 2008; 146:11-5. https://doi. org/10.1016/j.jss.2007.07.028.

23. Hsu WH, Hsu PK, Hsieh CC, Huang CS, Wu YC. The metastatic lymph node number and ratio are independent prognostic factors in esophageal cancer. J Gastrointest Surg. 2009; 13:1913-20. https://doi.org/10.1007/s11605-009-0982-8.

24. Edge SB, Compton CC. The American Joint Committee on Cancer: the 7th edition of the AJCC cancer staging manual and the future of TNM. Ann Surg Oncol. 2010; 17:1471-4. https://doi.org/10.1245/s10434-010-0985-4. 